\title{
Optimized Electron Bunch Current Distribution from a Radiofrequency Photo-Emission Source
}

\author{
Tianzhe Xu \\ Northern Illinois University \\ DeKalb, Illinois, U.S.A. \\ xu@niu.edu
}

\author{
Chunguang Jing \\ Euclid TechLab LLC. \\ Bolingbrook, Illinois, U.S.A. \\ c.jing@euclidtechlabs.com
}

\author{
Alexei Kanareykin \\ Euclid TechLab LLC. \\ Bolingbrook, Illinois, U.S.A. \\ alexkan@euclidtechlabs.com
}

\author{
Philippe Piot \\ Fermi National Accelerator Laboratory \& \\ Northern Illinois University \\ DeKalb, Illinois, U.S.A. \\ ppiot@niu.edu
}

\author{
John Power \\ Argonne National Laboratory \\ Lemont, Illinois, U.S.A. \\ jp@anl.gov
}

\begin{abstract}
In beam-driven acceleration, drive bunches with ramped current profiles support enhanced transformer ratios which enable longer interaction lengths thereby increasing the energy gain associated with the witness bunch. The production of such tailored electron bunches can be accomplished via different beam shaping methods. One technique of particular interest, owing to its simplicity, consists in shaping the temporal profile of the laser pulse in photoemission sources. In this contribution we investigate, via a shape-optimization technique, the generation of a ramped bunch distribution using such a lasershaping technique. Our study takes the example of the Argonne Wakefield Accelerator where a proof-of-principle experiment is being planned. We also discuss limitations associated with the laser shaping and possible improvements.
\end{abstract}

Keywords-collinear wakefield acceleration, beam shaping, photoinjector

\section{INTRODUCTION}

Beam-driven collinear wakefield acceleration is critical to the realization of compact accelerators in support, e.g., of future colliders and free-electron lasers. In this scheme, a high-charge bunch (the "drive" bunch) excites a wakefield while traveling through a high impedance structure. With proper configuration, this wakefield can be used to accelerate a trailing low-charge "witness" bunch [1-3]. A key parameter in beam-driven acceleration is the transformer ratio, which determines the energy gain of the witness bunch from the energy loss of the drive bunch. It is defined as the ratio between the maximum accelerating field behind the drive bunch and the maximum decelerating field within the drive bunch. Increasing the transformer ratio allows for the witness bunch to be accelerated to higher energy for a given drivebunch energy thereby lowering the cost of the drive-bunch beamline.

For drive bunches with symmetric current distribution, the transformer ratio is limited to values below 2 owing to the fundamental beam-loading theorem [4]. Larger

This work is supported by the U.S. Department of Energy contract No. DESC0017750 with Euclid Techlabs LLC., No. DE-SC0018656 with Northern Illinois University, and No. DE-AC02-06CH11357 with Argonne National Laboratory. transformer-ratio values can be attained with asymmetric bunch distribution [5] using beam shaping techniques. A sought-after shape is the linearly-ramped current profile where the current profile is a linear function of the bunch internal longitudinal coordinate $\zeta$ as $I(\zeta)=a(l-\zeta)+b$ for $\zeta \in[0, l]$ and $I(\zeta)=0$ elsewhere with $a, b$, and $l$ being constants. In our convention the head of the bunch corresponding to larger values of $\zeta$. To date there have been several methods capable of generating a ramped electron bunch [6], e.g. longitudinal phase space (LPS) correlation [7] and dielectric wakefield interaction [8]. Recently, a ramped bunch was demonstrated at Argonne Wakefield Accelerator (AWA) facility through an emittance-exchange beamline [9]. Among various shaping techniques, an appealing approach, applicable to the case of photoemission electron sources, consists in tailoring the photocathode laser temporal profile [10]. It is particularly attractive as it does not require any modification of the accelerator beamline. A pulse-shaping technique using birefringent crystals was recently reported at the photoinjector test facility at Deutsches Elektronen-Synchrotron, Zeuthen site for the enhancement of the transformer ratio in plasma wakefield acceleration [11]. There are also a few of other pulse-shaping methods based on optical instruments such as spatial light modulators (SLM) [12] and acousto-optic programmable dispersive filter (DAZZLER®) [13]. In the following, we describe the optimization of accelerator settings to obtain ramped electron bunch via photocathode-laser pulse shaping. Our optimization is applied to the AWA drive-beam photoinjector beamline [14] where an experiment is currently under consideration. We discuss limitations associated with this approach and possible mitigation techniques.

\section{Generation And Optimization of A RAMPed ELECTRON BUNCH}

\section{A. Photocathode Laser Shaping}

The laser shaping method that we plan to implement is based on a dispersive method using either an SLM or DAZZLER system. A simple model of the shaping 
process was elaborated within the SYNCHROTRON RADIATION WORKSHOP (SRW) framework [15]. Specifically, we consider an incoming spectrally-Gaussian laser beam with Gaussian transverse profile of the form $S(\omega, r)=A(\omega, r) e^{-\frac{\left(\omega-\omega_{0}\right)^{2}}{2 \sigma_{\omega}^{2}}} e^{-\frac{r^{2}}{2 \sigma_{r}^{2}}}$, where $\omega_{0}$ is the central frequency, $\sigma_{\omega}$ the spectral bandwidth and $\sigma_{r}$ the transverse beam size. The SRW simulations discretize the spectrum and transport each frequency component through a user-defined beamline. The temporal pulse shape downstream of the beamline can then be reconstructed via an inverse-Fourier transformation. To explore the laser-shaping technique, a new element was introduced in SRW and modelled as an arbitrary complex transmission function of the form $L(\omega, r)=T(\omega, r) e^{i H(\omega, r)}$ in the frequency domain as the laser pulse propagates through such an element its spectrum is transformed following $S(\omega, r) \rightarrow S^{\prime}(\omega, r)=L(\omega, r) S(\omega, r)$. In a first step, we ignore possible radial dependencies of the transmission function and take $L(\omega, r)=L(\omega)$. The code can then track the frequency-altered wavefronts in an arbitrary optical transport line as done in, e.g., Ref. [16]. Such an approach allows for the inclusion of spatiotemporal shaping. The simulation includes a chirp element with thickness $\delta z$, necessary to introduce a frequency-temporal chirp via chromatic effects using the transmission function $C(\omega)=e^{i k(\omega) \delta z}$ where $k(\omega)=k_{0}+\frac{\partial k}{\partial \omega}\left(\omega-\omega_{0}\right)+\frac{1}{2} \frac{\partial^{2} k}{\partial \omega^{2}}\left(\omega-\omega_{0}\right)^{2}+\mathcal{O}\left(\omega^{3}\right)$. In the simulations performed so far the spectrum amplitude was programmed to follow a ramp-like shape as exemplified in Fig. 1. Although the ultimate goal is to generate a linearly-
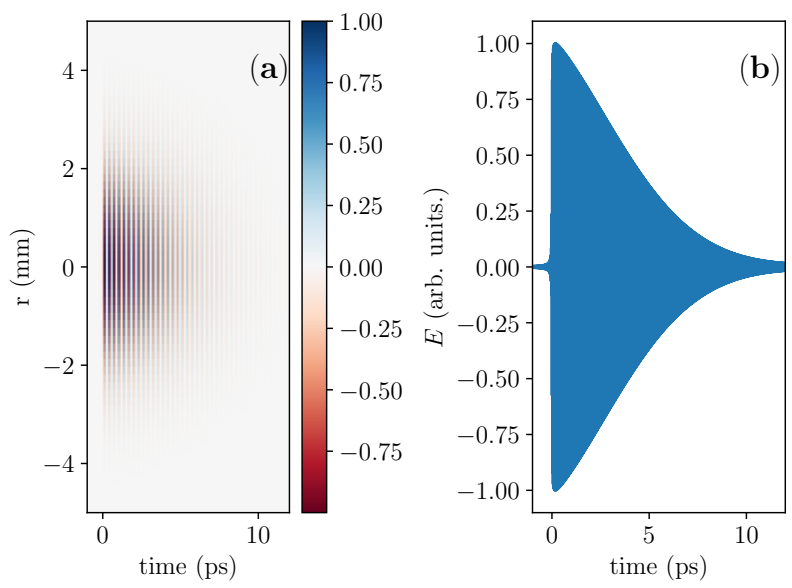

Fig. 1. Example spatiotemporal field associated with a ramped laser profile simulated with SRW (a) and corresponding on-axis $(r=0)$ intensitynormalized electric field. The head of the pulse is at larger times.

ramped electron-beam distribution some versatility in the laser-shaping process is needed. The SRW simulations are used to generate macroparticle distribution for beam-dynamics simulation. The simulated laser distribution is convolved in time with the photoemission response of the cathode. In our studies we consider the case of cesium telluride $\left(\mathrm{Cs}_{2} \mathrm{Te}\right)$ photocathodes empoyed at AWA given their high quantum efficiency. The response-time limitation is included using the parameterized time response of $\mathrm{Cs}_{2} \mathrm{Te}$ discussed in Ref. [17]. The macroparticle distribution is finally generated using a Monte-Carlo generator sampling the smoothed distribution.

\section{B. Optimization of the Electron-Bunch Shapes}

Owing to collective effects (space charge and image charge) and nonlinearities during the formation and early-stage beam dynamics, the laser-pulse temporal shape will not directly map into the electron-bunch current distribution. In general, and given the processes at play, the evolution of the longitudinal distribution is not easily described by an analytical model. Considering the formation of a linearly-ramped distribution at the end of photoinjector, we require an initial distribution that precompensates nonlinear distortions impressed during the emission process and beam evolution in the RF gun (where space-charge effects are prevalent). Following Ref. [18], we choose a laser-pulse shape of the form

$$
\rho(z)= \begin{cases}z^{\mu}, & \text { for } 0<z<1 \\ 0, & \text { elsewhere }\end{cases}
$$

where $z$ is the dimensionless longitudinal coordinate and the real exponent $\mu$ is to be determined by the optimization.

Given the complexity of the problem, we resort to optimization technique to devise the value of $\mu$, the laser spot radius and duration that produce the desired final bunch distribution. In order to quantitatively characterize the produced distribution after transport, two figures of merit are employed in the optimization. The skewness, which is customarily used in statistics to describe asymmetry of a distribution, is given by [19],

$$
S=\frac{1}{n} \sum_{i=1}^{n} \frac{\left(\zeta_{i}-\bar{\zeta}\right)^{3}}{\sigma_{\zeta}^{3}}
$$

where $\bar{\zeta}$ and $\sigma_{\zeta}$ are respectively the mean and standard deviation of longitudinal coordinate $\zeta$.

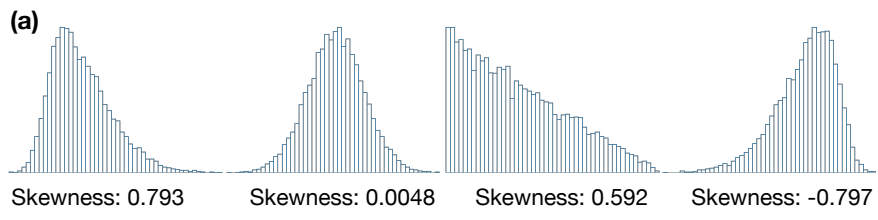

(b)
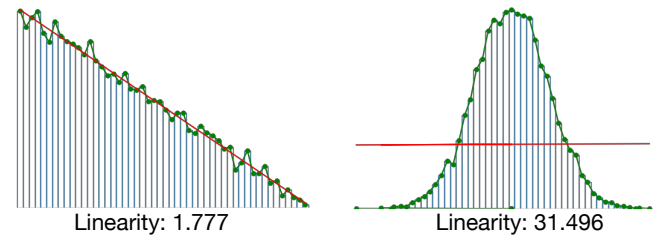

Fig. 2. (a) Skewness of a distribution for different bunch shapes. (b) Linearity of a triangular distribution and a Gaussian distribution

A second parameter - the linearity - measures the smoothness of the linear ramp. The LPS distribution $\Phi(\zeta, \delta)$ (here $\zeta$ 
and $\delta$ are respectively the longitudinal position and fractional momentum offset w.r.t. the bunch's barycenter) and obtained downstream of the last linac cavity (C6) was longitudinally binned (over bins with width $\delta \zeta$ ) to yield the histogram function $H_{i}=\int_{\zeta_{i}-\Delta \zeta / 2}^{\zeta_{i}+\Delta \zeta / 2} d \zeta \int_{-\infty}^{+\infty} d \delta \Phi(\zeta, \delta)$. The linearity parameter is then computed as,

$$
L \equiv \frac{1}{n} \sum_{i=1}^{n}\left|H_{i}\left(\zeta_{i}\right)-f\left(\zeta_{i}\right)\right|
$$

where a linear regression was performed on $H_{i}\left(\zeta_{i}\right)$ thereby yielding a fitted polynomial $f(\zeta)=a+b \zeta$. Therefore the linearity parameter represent an average distance between the fitted line and binned data. Fig. 2 illustrate values of the skewness and linearity for a Gaussian and linear-ramped distribution. A linearly-ramped distribution as needed for beam-driven acceleration is expected to have $(i)$ a positive skewness and $(i i)$ a small linearity.

\section{Start-TO-END Simulations}

We performed beam-dynamics simulations to explore the formation of a linearly-ramped bunches using the AWA drivebeam photoinjector diagrammed in Fig. 3. The beamline incorporates a $1+1 / 2 \mathrm{~L}$-band $(1.3 \mathrm{GHz}) \mathrm{RF}$ gun followed by six 7-cell cavities. The RF-gun cavity is surrounded by three solenoidal lenses and includes a $\mathrm{Cs}_{2}$ Te photocathode which is impinged by an ultraviolet laser pulse obtained via frequency tripling of an amplified infrared laser. In our simulation two of the linac cavities ( $\mathrm{C} 4$ and $\mathrm{C} 6$ ) were turned off to mimic one of the standard-operating configurations.

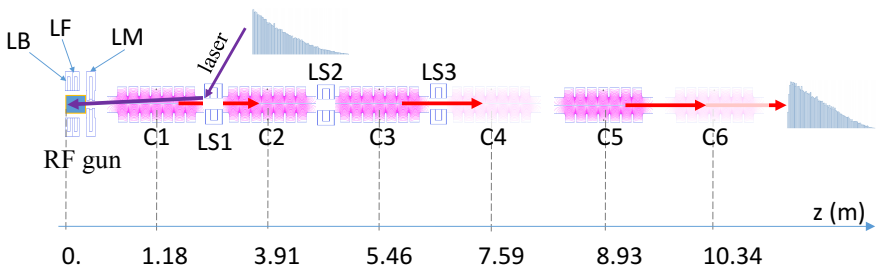

Fig. 3. Layout of the AWA drive-beam photoinjector beamline. The $\mathrm{C} i$ labels refer to accelerating cavity [the shaded cavities ( $\mathrm{C} 4$ and $\mathrm{C} 6$ ) were turned off in our simulations]. L $i$ are solenoidal lenses.

The beam was modeled with IMPACT-T [20], a particlein-cell beam-dynamics program that includes 3D spacecharge effect. The multi-objective optimization was implemented within the DEAP [21] evolutionary computation framework. Our implementation includes a parallelization mechanism using the SCOOP multiprocessing toolbox [22]. Specifically, we used the fast-elitist non-dominated sorting genetic algorithm commonly employed in multi-objective optimizations [23]. Aside from the exponent $\mu$ of the initial distribution (Eq. (1)), nine parameters were varied: field strength of matching solenoid (LM) and linac solenoids (LS1, LS2 and LS3), the laser launch phase $\left(\phi_{\text {laser }}\right)$, and accelerating-cavity phases $\left(\phi_{1}, \phi_{2}, \phi_{3}, \phi_{5}\right)$. The minimized objectives were the emittance and linearity parameter. The
TABLE I

Accelerator Settings and Final Simulated Beam Parameters Associated With One SeT Of Optimized PaRAMETERS.

\begin{tabular}{lrr}
\hline Parameter & Value & units \\
\hline Number of macroparticles & 200,000 & - \\
\hline Bunch charge & 3 & $\mathrm{nC}$ \\
\hline Emission time & 30 & $\mathrm{ps}$ \\
\hline Exponent $\mu$ & 1.14 & \\
\hline Laser spot radius & 2 & $\mathrm{~mm}$ \\
\hline RF gun peak E field & 35 & $\mathrm{MV} / \mathrm{m}$ \\
\hline laser launch phase & 22 & $\mathrm{deg}$ \\
\hline C1,2,3,5 cavity E field & $10,12,12,13$ & $\mathrm{MV} / \mathrm{m}$ \\
\hline $\mathrm{C} 1,2,3,5$ cavity off-crest phase & $50,11,25,17$ & $\mathrm{deg}$ \\
\hline Final beam energy & 43 & $\mathrm{MeV}$ \\
\hline Final transverse emittance & 2.1 & $\mu \mathrm{m}-\mathrm{rad}$ \\
\hline Final RMS bunch length & 2.4 & $\mathrm{~mm}$ \\
\hline
\end{tabular}

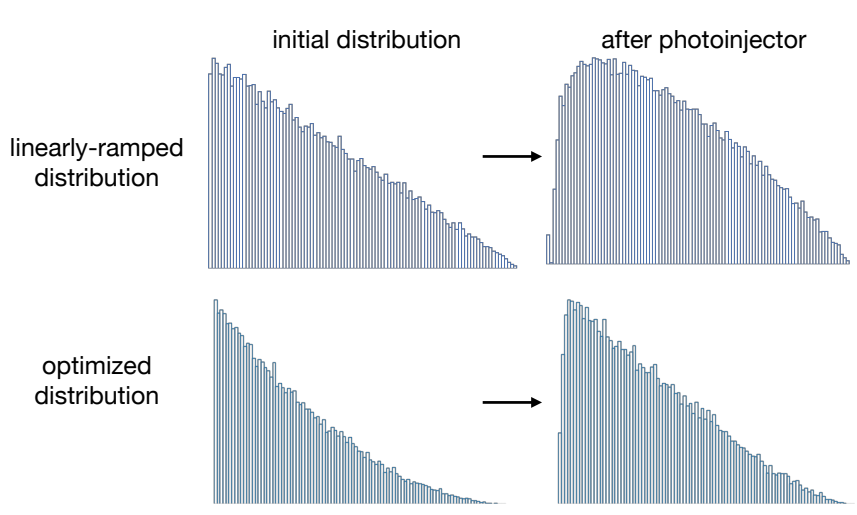

Fig. 4. Example of achieved final current distribution (right) with associated initial laser distribution (left). The upper and lower row respectively corresponds to a linearly-ramped and power-like (Eq. (1) with $\mu=2$ ) initial distributions.
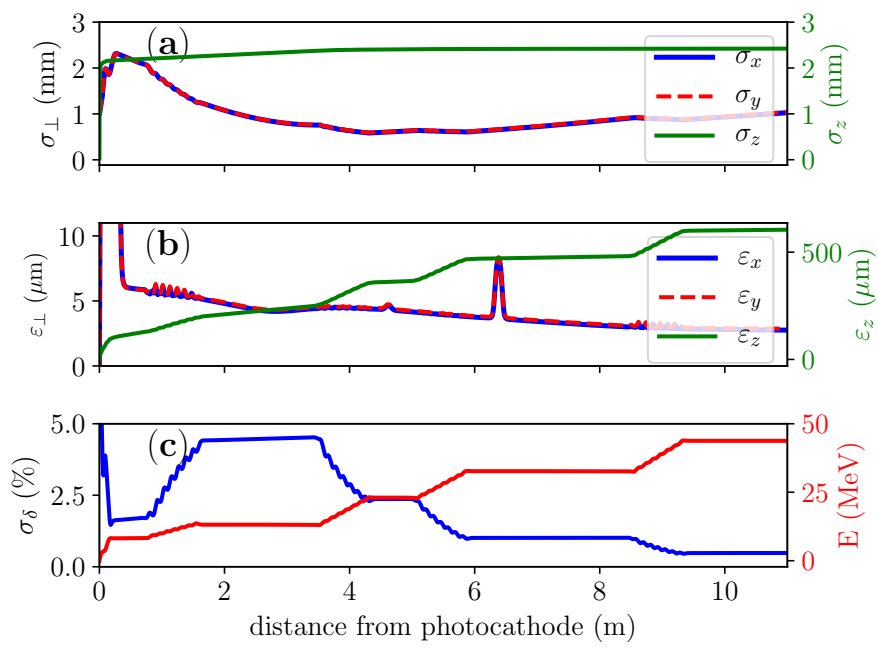

Fig. 5. Evolution of the transverse $\sigma_{\perp}$ and longitudinal $\sigma_{z}$ beam sizes, transverse $\varepsilon_{\perp}$ and longitudinal $\varepsilon_{\perp}$ emittances (b) and energy $E$ and fractional RMS energy spread $\sigma_{\delta}$ along the beamline with parameters optimized to realize a linearly-ramped current profile.

skewness parameter was introduced as a constraint to select asymmetric distributions while kinetic energy was added to control the diversity of the population (only individuals with 
kinetic energy $K>40 \mathrm{MeV}$ were retained).

The final bunch current profiles achieved for two initial laser distributions appear in Fig. 4. As can be seen, an initially linearly-ramped laser distribution evolve into a "bulging" distribution with a strong quadratic distortion, whereas an optimized distribution with $\mu=2$ "blows out" into a linearlyramped current profile at the end of photoinjector.
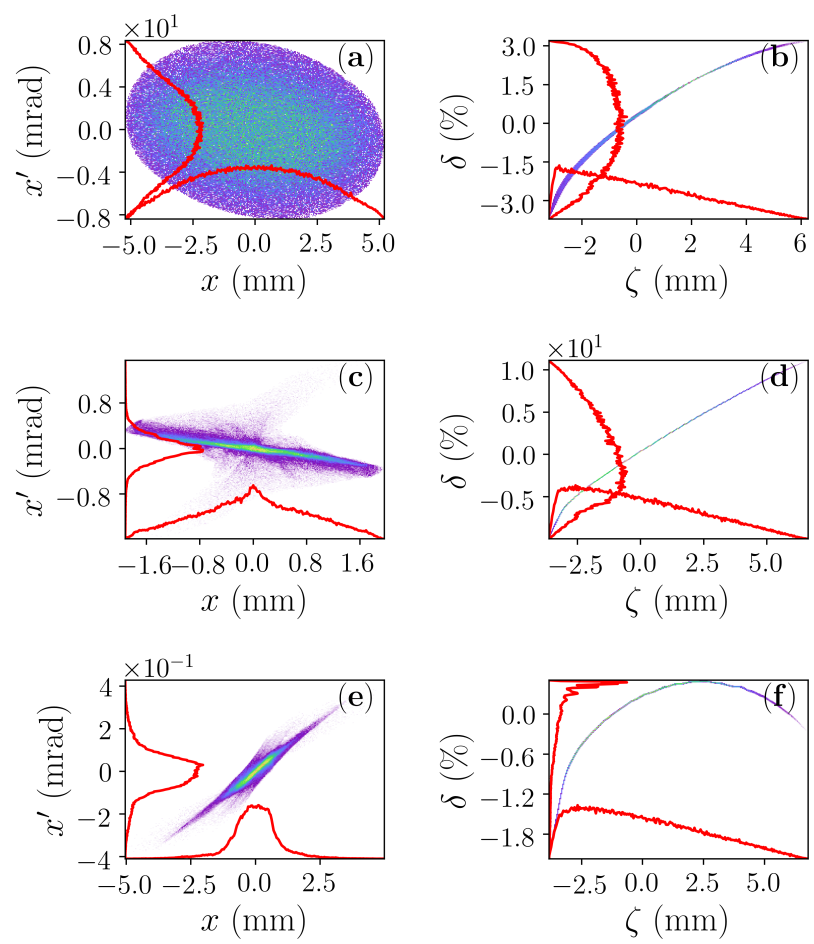

Fig. 6. Transverse (a, c, e) and longitudinal (b, d, f) phase space snapshots downstream of the RF gun $(a, b)$, cavity $\mathrm{C} 1(\mathrm{c}, \mathrm{d})$, and cavity $\mathrm{C} 6(\mathrm{e}, \mathrm{f})$. The head of the bunch corresponds to $\zeta>0$.

An example of beam parameters obtained downstream of the linac is summarized in Table I together with some of the associated accelerator settings. Fig. 5 displays the evolution of critical beam parameters along the beamline. Although a transverse beam emittance of $\sim 2.1 \mu \mathrm{m}$ is attained, it should be noted that the initial laser pulse duration is taken to be rather long ( $30 \mathrm{ps}$ full width in this set of optimizations). In addition, as can be seen on the sequence of phase space presented in Fig. 6, the LPS acquires a large quadratic distortion owing to the initial long bunch length and relatively-large bunch charge $Q=3 \mathrm{nC}$. The later effect could eventually be corrected via deceleration in a harmonic accelerating cavity or using wakefield [24].

\section{CONCLusions}

From the presented results we simulated the production of a ramped electron bunch from the photocathode. These preliminary results especially support the feasibility of generating a ramped electron bunch via a photocathode-laser shaping method at the AWA beamline.

However, photocathode laser shaping provides limited capability to produce short bunches as the bunch length is limited by the initial duration of the laser pulse and collective effects arising during the low-energy transport. The formation of a $\mathrm{GeV}$-class drive bunch for beam-driven collinear wakefield acceleration will consequently rely on a multi-stage bunchcompression scheme to attain a final bunch length consistent with the wavelength of the wakefield to be excited in the beamdriven accelerator. During the acceleration to higher energies and the compression process, the bunch will experience other collective effects such as coherent synchrotron radiation, longitudinal space charge, and wakefield, which will most likely alter the LPS and ultimately affect the current profile. Therefore, more comprehensive start-to-end simulations including the full LPS-manipulation process will be needed to fully optimize the proposed technique.

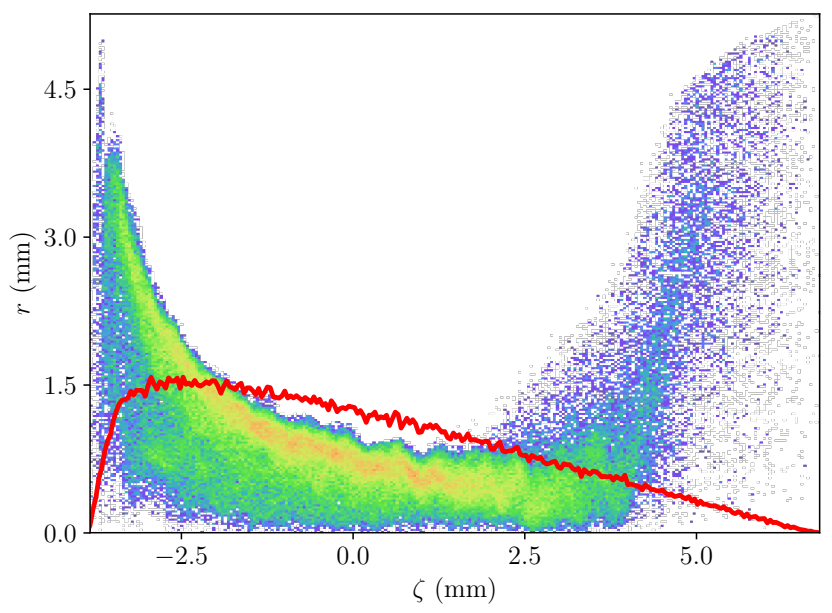

Fig. 7. Spatiotemporal $(\zeta, r)$ distribution obtained downstream of the AWA linac [this corresponds to the data shown in Fig. 6(e-f)]. The radius is defined as $r \equiv \sqrt{x^{2}+y^{2}}$.

Another limitation of the laser shaping scheme is the distortion of bunch shapes in the space-charge dominated regime. In the case of a high-charge bunch or low-gradient gun, the electron bunch will morph under the effect of space charge force. A possible way of compensating such effect is through further optimization of initial bunch shapes, e.g., by replacing Eq. (1) with a higher-power polynomial of the form $\rho(z)=\sum_{i=0}^{N} a_{i} z^{\mu_{i}}$. Additionally, the simulated final beam distribution displays some nonlinear correlation in the $(\zeta, r)$ space. We especially find that the beam size $\sigma_{r}$ varies strongly along the bunch longitudinal coordinate; see Fig. 7. Producing distributions without such a correlation could be accomplished by introducing a transmission function with radial dependence. A possible solution would be the generation of a tailored laser profile with a time dependent radius to produce larger radius in the (higher-intensity) tail region of the laser thereby providing 
a constant current-density bunch during the emission process.

Finally, the preservation of a sharp edge, especially on the tail side, is critical to beam-driven acceleration (a smearing of the edge results in an increased energy spread for the drive bunch as it is decelerated in the wakefield-acceleration process). Such sharp edge will most likely have to be realized at high energy, once the longitudinal dynamics is frozen, using dispersive collimation [25] or possibly phase-space exchanging beamlines [26].

\section{REFERENCES}

[1] P. Chen, J. M. Dawson, R. W. Huff, and T. Katsouleas Phys. Rev. Lett., vol. 54, pp. 693-696, Feb 1985.

[2] G. A. Voss and T. Weiland, "Particle acceleration by wakefields," DESY Hamburg Report No. DESY-M-82-10, 1982.

[3] W. Gai, P. Schoessow, B. Cole, R. Konecny, J. Norem, J. Rosenzweig, and J. Simpson, "Experimental demonstration of wake-field effects in dielectric structures," Phys. Rev. Lett., vol. 61, pp. 2756-2758, Dec 1988.

[4] R. D. Ruth, P. Morton, P. B. Wilson, and A. Chao, "A plasma wake field accelerator," Part. Accel., vol. 17, no. SLAC-PUB-3374, p. 171, 1984.

[5] K. L. Bane, P. Chen, and P. Wilson, "On collinear wake field acceleration," Report No. SLAC-PUB-3662, 1985.

[6] P. Piot, C. Behrens, C. Gerth, M. Dohlus, F. Lemery, D. Mihalcea, P. Stoltz, and M. Vogt, "Generation and characterization of electron bunches with ramped current profiles in a dual-frequency superconducting linear accelerator," Phys. Rev. Lett., vol. 108, p. 034801, Jan 2012.

[7] P. Piot, Y.-E. Sun, J. G. Power, and M. Rihaoui, "Generation of relativistic electron bunches with arbitrary current distribution via transverseto-longitudinal phase space exchange," Phys. Rev. Spec. Top.-Accel. Beams, vol. 14, p. 022801, Feb 2011.

[8] G. Andonian, S. Barber, F. H. O'Shea, M. Fedurin, K. Kusche, C. Swinson, and J. B. Rosenzweig, "Generation of ramped current profiles in relativistic electron beams using wakefields in dielectric structures," Phys. Rev. Lett., vol. 118, p. 054802, Feb 2017.

[9] G. Ha, M. H. Cho, W. Namkung, J. G. Power, D. S. Doran, E. E. Wisniewski, M. Conde, W. Gai, W. Liu, C. Whiteford, Q. Gao, K.-J. Kim, A. Zholents, Y.-E. Sun, C. Jing, and P. Piot, "Precision control of the electron longitudinal bunch shape using an emittance-exchange beam line," Phys. Rev. Lett., vol. 118, no. 10, p. 104801, 2017.

[10] F. Lemery and P. Piot, "Tailored electron bunches with smooth current profiles for enhanced transformer ratios in beam-driven acceleration," Phys. Rev. Spec. Top.-Accel. Beams, vol. 18, no. 8, p. 081301, 2015.

[11] G. Loisch, G. Asova, P. Boonpornprasert, R. Brinkmann, Y. Chen, J. Engel, J. Good, M. Gross, F. Grüner, H. Huck, D. Kalantaryan, M. Krasilnikov, O. Lishilin, A. M. de la Ossa, T. J. Mehrling, D. Melkumyan, A. Oppelt, J. Osterhoff, H. Qian, Y. Renier, F. Stephan, C. Tenholt, V. Wohlfarth, and Q. Zhao, "Observation of high transformer ratio plasma wakefield acceleration," Phys. Rev. Lett., vol. 121, p. 064801, Aug 2018.

[12] A. M. Weiner, "Femtosecond pulse shaping using spatial light modulators," Rev. Sci. Instrum., vol. 71, no. 5, pp. 1929-1960, 2000

[13] P. Tournois, "Acousto-optic programmable dispersive filter for adaptive compensation of group delay time dispersion in laser systems," Opt. Commun., vol. 140, no. 4-6, pp. 245-249, 1997.

[14] M. Conde and et al., "Research program and recent results at the argonne wakefield accelerator facility," Proceedings of the 8th International Particle Accelerator Conference, Copenhagen, Denmark, pp. 2885-2887, 2017.

[15] O. Chubar, "Wavefront calculations," Proc. SPIE, X-Ray FEL Optics and Instrumentation, vol. 4143, pp. $4143-4143-12,2001$.

[16] M. Andorf, V. Lebedev, P. Piot, and J. Ruan, "Wave-optics modeling of the optical-transport line for passive optical stochastic cooling," Nucl. Instrum. Methods Phys. Res., Sect. A, vol. 883, pp. 166-169, 2018.

[17] G. Ferrini, P. Michelato, and F. Parmigiani, "A Monte Carlo simulation of low energy photoelectron scattering in $\mathrm{Cs}_{2} \mathrm{Te}$," Solid State Commun., vol. 106 , no. 1 , pp. 21-26, 1998
[18] F. Lemery and P. Piot, "Temporal electron-bunch shaping from a photoinjector for advanced accelerator applications," Proceedings of the 5th International Particle Accelerator Conference, Dresden, Germany, p. 1454, 2014.

[19] S. Kokoska and D. Zwillinger, CRC standard probability and statistics tables and formulae. Crc Press, 1999.

[20] J. Qiang, S. Lidia, R. D. Ryne, and C. Limborg-Deprey, "Threedimensional quasistatic model for high brightness beam dynamics simulation," Phys. Rev. Spec. Top.-Accel. Beams, vol. 9, p. 044204, Apr 2006.

[21] F.-A. Fortin, F.-M. De Rainville, M.-A. Gardner, M. Parizeau, and C. Gagné, "DEAP: Evolutionary algorithms made easy," Journal of Machine Learning Research, vol. 13, pp. 2171-2175, jul 2012.

[22] Y. Hold-Geoffroy, O. Gagnon, and M. Parizeau, "Once you scoop, no need to fork," in Proceedings of the 2014 Annual Conference on Extreme Science and Engineering Discovery Environment, p. 60, ACM, 2014.

[23] K. Deb, A. Pratap, S. Agarwal, and T. Meyarivan, "A fast and elitist multiobjective genetic algorithm: Nsga-ii," IEEE Transactions on Evolutionary Computation, vol. 6, no. 2, pp. 182-197, 2002.

[24] P. Craievich, "Passive longitudinal phase space linearizer," Phys. Rev. Spec. Top.-Accel. Beams, vol. 13, p. 024401, 2010.

[25] P. Muggli, V. Yakimenko, M. Babzien, E. Kallos, and K. K. P., "Generation of trains of electron microbunches with adjustable subpicosecond spacing," Phys. Rev. Lett., vol. 101, p. 054801, 2008.

[26] Y. Sun, P. Piot, A. Johnson, A. H. Lumpkin, T. J. Maxwell, J. Ruan, and R. Thurman-Keup, "Subpicosecond electron-bunch-train generation using a transverse-to-longitudinal phase-space exchange technique," Phys. Rev. Lett., vol. 105, p. 054801, 2010. 